\title{
Nano Brazing of Pt-Ag Nanoparticles under Femtosecond Laser Irradiation
}

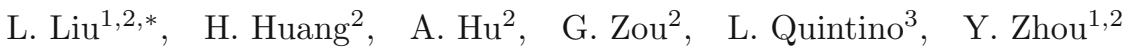

(Received 19 January 2013; accepted 2 April 2013; published online 10 April 2013)

\begin{abstract}
Nano brazing of Pt-Ag nanoparticles with nano Ag filler metal is reported in this letter, which presents an effective way to join nanoobjects by femtosecond laser irradiation. The nano brazed interface

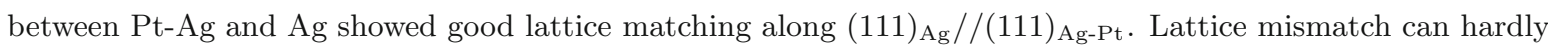
be observed at the interface between the filler metal and Pt-Ag nanoparticle, which is important for the joint strength and normally does not occur during joining. The very low mismatch also suggested that melting and solidification occurred during nano brazing by femtosecond laser. The role of Brownian motion on the nano joining process is also discussed in this paper.
\end{abstract}

Keywords: Femtosecond laser; Nanojoining; Nanoparticles

Citation: L. Liu, H. Huang, A. Hu, G. Zou, L. Quintino and Y. Zhou, "Nano Brazing of Pt-Ag Nanoparticles under Femtosecond Laser Irradiation", Nano-Micro Lett. 5(2), 88-92 (2013). http://dx.doi.org/10.5101/ $\mathrm{nml} \cdot \mathrm{v} 5 \mathrm{i} 2 \cdot \mathrm{p} 88-92$

\section{Introduction}

Further down the road of miniaturization to nanoscale, there is an emerging need to join nanoobjects to form nanoscale devices and systems [1-3]. Nanoparticles are regarded as important parts of nanoscale devices, which make joining nanoparticles necessary as a basic integration process in nanoscale products. It is still challenging to interconnect single nanoobjects with a stable Ohmic contact, which is a key issue for nanoelectronic device manufacturing $[1,4]$. Direct "fusion" joining of nanoobjects is one way to achieve stable conductive nanojunctions $[5,6]$. However, most of the "fusion" nanojoining methods are applied for making nanojunctions between nanowires $[7,8]$, because it is hard to control the melting depth of nanoparticles [1]. The consequence is that when two nanoparticles are totally melted, the very high surface tension at nanoscale would merge the two individual nanopar- ticles into one larger nanoparticle [9].

In this regard, nano brazing/soldering needs to be developed for the purpose of joining nanoparticles with high Ohmic conductivity, since brazing can produce both minimum damage to the base metal and a desired joint morphology. Use of a femtosecond laser can result in an ultrafast and nonthermal melting of solid materials with precisely controlled energy, which is promising for developing nano brazing/soldering junctions for nano- and/or molecular devices [2]. Only a few research works have focused on this area [5], since most of the nanojoining processes were only developed in the past decade and the concept of nanojoining was just proposed very recently [2]. In the present study, an effective and controlled manner of using a femtosecond laser to produce nano brazing joints was demonstrated and the joints were characterized in detail by transmission electron microscope (TEM).

\footnotetext{
${ }^{1}$ Department of Mechanical Engineering, Tsinghua University, Beijing, 100084, China

${ }^{2}$ Department of Mechanical \& Mechatronics Engineering, University of Waterloo, Waterloo, N2L 3G1 Canada

${ }^{3}$ Technical University of Lisbon, 2740-120 Porto Salvo, Portugal

*Corresponding author. E-mail: liulei@tsinghua.edu.cn
} 


\section{Experiment}

A Ti-sapphire laser system (Coherent Inc) was operated to generate 35 fs laser pulses at repetition rate of $1 \mathrm{kHz}$ and $800 \mathrm{~nm}$. Each laser profile was confirmed to be Gaussian with output energy of $2 \mathrm{~mJ} /$ pulse. Laser pulses were focused in the middle of a 2-ml-glass quartz vessel by a lens with $13 \mathrm{~cm}$ focal length. First, surfactant-free platinum nanoparticles in pure ethanol were prepared by laser ablation of $\mathrm{Pt}$ rods for $3 \mathrm{~min}$ at the focal point. The laser ablation of $\mathrm{Pt}$ in ethanol was accompanied by the presence of a plasma plume on the target surface, easily visible by naked eye. A visible coloration of the solution was observed after the experiment. Ablation of $\mathrm{Ag}$ was performed right after $\mathrm{Pt}$ in the same solution. The Ag rod was defocused by $5 \mathrm{~mm}$ and ablated for $30 \mathrm{~min}$. The nanoparticles' composition, identification, joint morphology and the nano brazing interface were examined by a field emission transmission electron microscope (TEM, product of JEOL JEM-2010F) operated at both high resolution (HRTEM) and high-angle annular dark field scanning TEM (STEM) mode.

To our knowledge, most laser nanoalloying or nanojoining of nanoparticles using a femtosecond laser is a two step procedure: mixing two prepared pure metal nanocolloid solutions and then irradiating the mixed solution by laser [10, 11]. In our experiment mixing and irradiating occurred at the same time. This not only improves efficiency, but also facilitates the experimental design for three reasons. First, the laser energy density distribution in the glass vessel was significantly non-uniform because of the Gaussian beam and focusing lens. This tended to increase the uncertainty and uncontrollability. With our method the interaction between $\mathrm{Ag}$ and Pt-Ag nanoparticles occurred at a controllable place, which was the space in front of the bulk Ag rod. Second, the silver nanoparticles were gradually synthesized during the $30 \mathrm{~min}$. This means the ratio of $\mathrm{Ag}$ to $\mathrm{Pt}$ increased with irradiation time, suggesting that the number of $\mathrm{Pt}$ nanoparticles surrounding the $\mathrm{Ag}$ nanoparticles was fairly large at the beginning, which expected to increase the chance of interaction between dissimilar nanoparticles. Third, low energy ablation can generate tiny nanoparticles (less than $10 \mathrm{~nm}$ ) [12], which can dramatically reduce the melting temperature of the silver nano brazing filler metal [9].

\section{Results and discussion}

Figure 1(a) shows STEM image of two typical individual nanoparticles, the left one was $\mathrm{Ag}$ and the right one was Pt-Ag nanoalloy, which were confirmed by EDX line scanning as shown in Fig. 1(b). The insert pictures in Fig. 1(a) are HRTEM images of the two nanoparticles in Fig. 1(a). The formation of PtAg nanoparticles was similar to the laser nanoalloying process which can be found elsewhere $[10,13]$. The STEM image in Fig. 1 indicates that the element distribution of the Pt-Ag nanoparticle was not uniform. However, in HRTEM mode the lattice spacing of the $\mathrm{Pt}-\mathrm{Ag}$ nanoparticle was the same.
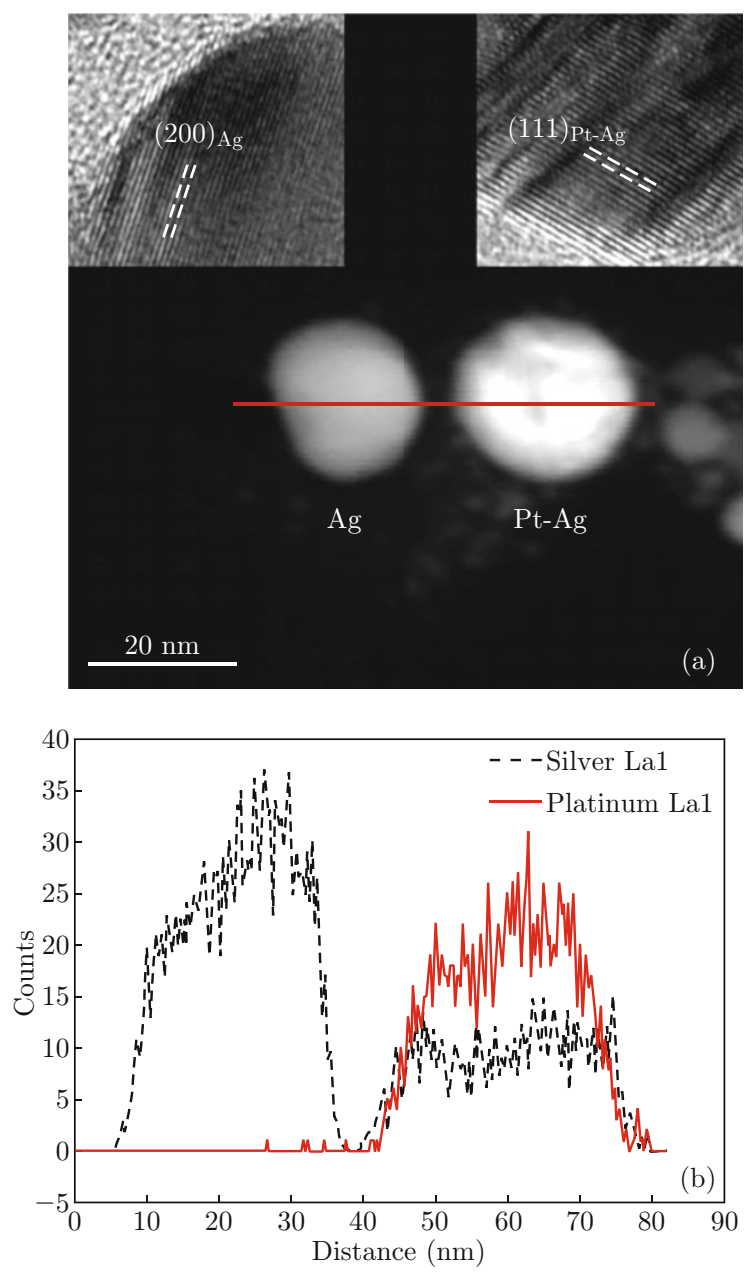

Fig. 1 Typical Ag and Pt-Ag nanoparticles: (a) STEM of isolated $\mathrm{Ag}$ and $\mathrm{Pt}-\mathrm{Ag}$ nanoparticles, the lattice spacing of $(200)_{\mathrm{Ag}}$ was $2.09 \AA$ and $(111)_{\mathrm{Pt}-\mathrm{Ag}}$ was $2.33 \AA$; (b) EDS line scan in (a).

The measured lattice spacing for $\mathrm{Ag}$ was $(200)_{\mathrm{Ag}}=$ $2.09 \AA$ while Pt-Ag alloy $(111)_{\mathrm{Pt}-\mathrm{Ag}}=2.33 \AA$. The lattice parameter of laser induced $\mathrm{Pt}-\mathrm{Ag}$ nanoalloy was the same as in ref. [14], which was prepared by wet chemical method. The lattice constants calculated based on the measurements were $a=4.18 \AA$ for $\mathrm{Ag}$ and $a=4.04$ $\AA$ for Pt-Ag. The measured lattice constant of $\mathrm{Ag}$ was $3.5 \%$ larger than bulk Ag which may be due to the size dependent lattice parameter and apparatus error [4]. The lattice mismatch between the Ag and Pt-Ag can 
be calculated by Equation 1

$$
\Delta a=\frac{\left|a_{1}-a_{2}\right|}{\frac{1}{2}\left(a_{1}+a_{2}\right)}
$$

in which, $\Delta a$ is mismatch, $a_{1}$ and $a_{2}$ are lattice parameters of two materials. The calculation shows that the theoretical mismatch between $\mathrm{Ag}$ and Pt-Ag nanoalloy was less than $2 \%$.

Typical joined spherical nanoparticles are shown in Fig. 2 in STEM mode. The image clearly shows that the interjunction between spherical $\mathrm{Pt}-\mathrm{Ag}$ nanoparticles was made by nano-Ag. Due to the low laser fluence, the size of the nano Ag filler metal was less than $10 \mathrm{~nm}$, having the melting temperature in the range of 373-573 $\mathrm{K}$ [15], which is much lower than that of Pt. Small radius fillets were found at the braze joint, but not a well-developed wetting over the Pt-Ag surface which can be found in brazing of bulk material. This is due to the ultrafast cooling rate and limited time for the melted Ag to completely wet and spread. Since both $\mathrm{Pt}$ and $\mathrm{Ag}$ have very low bulk resistance, and the
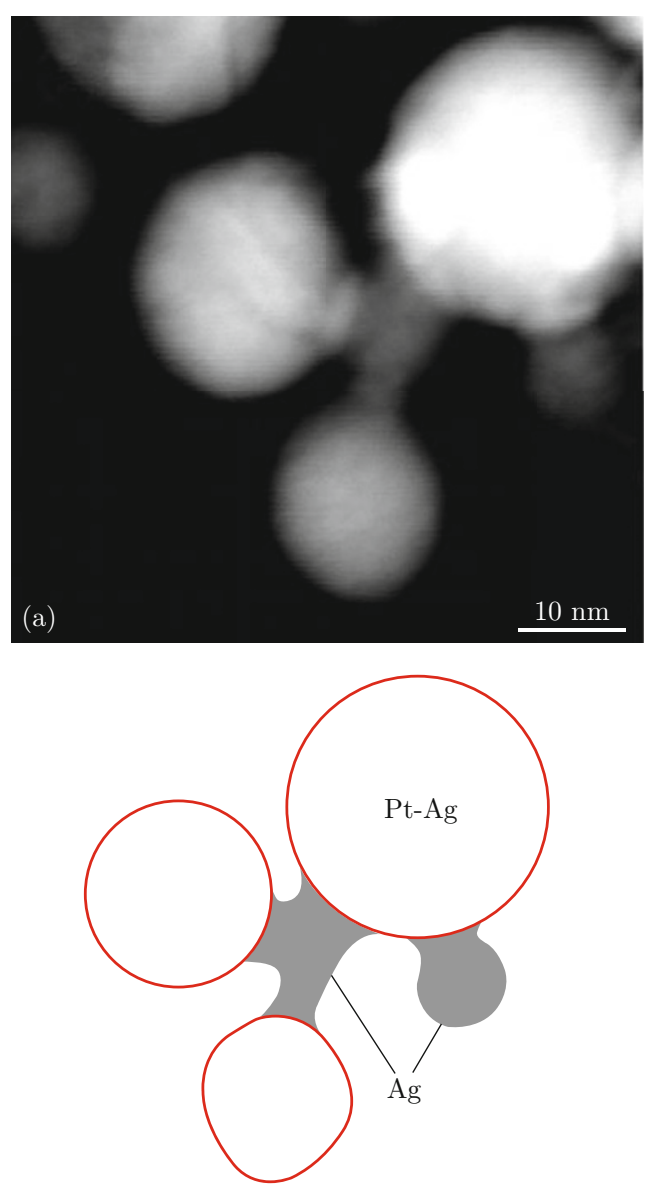

(b)

Fig. 2 STEM image of nano brazed joint by femtosecond laser irradiation. The nano Ag cluster was the filler metal and Pt-Ag nanoalloy was the base metal. brazed interfaces showed atomic scale bonding and no intermetallic compounds formed, it is believed that the contact (or joint) had good electric conductivity. This structure leads us to conclude that the in-situ synthesized Ag nanoparticles acted as nano filler metal which created ohmic joints among Pt-Ag nanoparticles.

It is believed that the nano brazing process is also related to the Brownian motion. At very small particle size, as in nanofluids, Brownian motion becomes important [16]. The root-mean-square velocity $\left(\nu_{N}\right)$ of a Brownian particle can be defined as [16]

$$
v_{N}=\sqrt{\frac{3 k_{b} T}{m_{N}}}=\frac{1}{d_{N}} \sqrt{\frac{18 k_{b} T}{\pi \rho_{N} d_{N}}}
$$

where $k_{b}$ is the Boltzmann constant, $T$ the temperature, $m_{N}$ the particle mass, $\rho_{N}$ the density and $d_{N}$ the diameter of the nanoparticle. According to the classic collision theory, the collision frequency is

$$
Z=N_{A} \sigma_{A B} \sqrt{\frac{8 k_{b} T}{\pi \mu_{A B}}}
$$

where $N_{A}$ is the Avogadro constant, $\sigma_{A B}$ the reaction cross section, $k_{b}$ the Boltzmann's constant, and $\mu_{A B}$ the reduced mass of the reactants. In the nanofluid theory, an enhanced thermal conductivity of nanoscale colloidal solutions has been found which is primarily due to convection caused by the Brownian movement of these nanoparticles [16]. Collision between nanoparticles is also one of the reasons for the enhanced thermal conductivity but it can be neglected according to order-of-magnitude analysis [17]. In contrary, the role of collision between nanoparticles is very important to a joining process because the two nanoparticles should be in contact before brazing/joining [5] and the contact is believed primarily caused by collision. When collision and laser irradiation occur at the same time, joining tends to occur due to the non-thermal ultrafast melting phenomenon of femtosecond laser. When collision occurs but no laser irradiation, the nanoparticles may also contact because contact can lower the free energy of the system. However, in this case the contacted area would be limited by the free energy reduction value. Following the contact, when laser irradiates, joining also tends to occur and results in well-wetted brazing joint. Therefore, it is believed that both Brownian movement and surface energy played important roles in the nano brazing process.

Figure 3 shows the orientation relationship of the nano brazing interface between $\mathrm{Ag}$ and $\mathrm{Pt}-\mathrm{Ag}$ nanoalloy which were in the same zone axis. The upper-left insert STEM image indicates the interface between $\mathrm{Ag}$ and Pt-Ag nanoalloy. HRTEM of the same position shows that $(111)_{\mathrm{Ag}} / /(111)_{\mathrm{Ag}-\mathrm{Pt}}$ and the mismatch between $\mathrm{Ag}$ and $\mathrm{Pt}-\mathrm{Ag}$ can hardly be observed. The lattices at the interface matched very well, as needed to achieve a 
good joint strength, especially for a heterophase interface [18]. Usually, this does not occur because lattice defects at the brazing interface occurred in most cases because of either lattice constant mismatch or residual stress induced by the brazing process. We believe that there were two reasons for the very low mismatching. First, the lattice constant of $\mathrm{Ag}$ and $\mathrm{Pt}-\mathrm{Ag}$ was nearly the same, as calculated above based on the lattice image in Fig. 1(a). Second, a melting and epitaxial growth (or solidification) process occurred during femtosecond laser irradiation. For large nanoparticles, only surface melting occurs during femtosecond laser irradiation $[19,20]$. The Ag nano brazing filler cluster was very small (normally less than $10 \mathrm{~nm}$ ) due to the lower ablation energy used. If nanoparticles are tiny clusters with limited atoms, they may suddenly display global melting without surface melting, because the effective laser penetration depth was larger than the particle size [19]. After the laser pulse turned off, the Ag nano brazing filler metal solidified and grew epitaxially on the Pt-Ag surface.

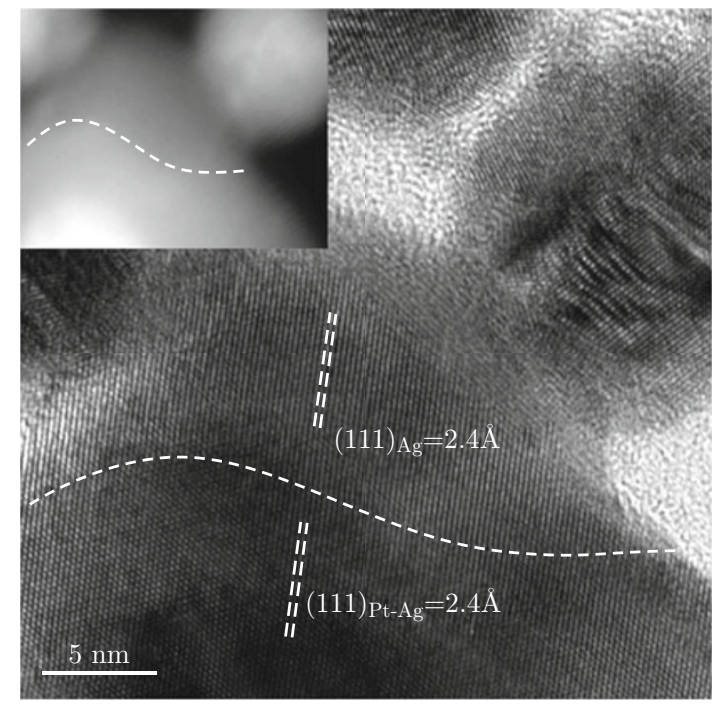

Fig. 3 Orientation relationship between Ag-Pt nanoalloy and Ag nano-brazing filler metal, showing the minimum interfacial mismatch.

\section{Conclusion}

This study presents an effective way to join nanoobjects by femtosecond laser brazing, in which femtosecond laser was used to generate tiny nano-Ag clusters as brazing filler metal and nano brazed Pt-Ag nanoparticles. The nano brazed interface between Pt-Ag and $\mathrm{Ag}$ showed good lattice matching along (111) $\mathrm{Ag} / /$ $(111)_{\mathrm{Ag}-\mathrm{Pt}}$. It suggests that melting and solidification occurred during femtosecond laser nano brazing. The nano-networks of $\mathrm{Pt}$-Ag produced by femtosecond irradiation make them very attractive for several applications, such as plasmonics, catalysts and nanoscale elec- tric devices.

\section{Acknowledgements}

This work was supported by the Canadian Research Chairs (CRC) program, by National Sciences and Engineering Research Council (NSERC), State Key Lab of Advanced Welding \& joining, HIT (No. AWPT-Z12-04) National Natural Science Foundation of China (Grant No. 51075232) and Tsinghua University Initiative Scientific Research Program (Grant No. 2010THZ 02-1). The authors would like to thank Mr. Fred Pearson from McMaster University, for help with TEM. Appreciation is also expressed to Prof. Scott Lawson for useful discussions.

\section{References}

[1] S. Guo, "The creation of nanojunctions", Nanoscale 2(12), 2521-2529 (2010). http://dx.doi.org/10. 1039/CONR00352B

[2] Y. Zhou and A. Hu, "From microjoining to nanojoining", Open Surf. Sci. J. 3, 32-41 (2008).

[3] C. Chen, Y. Lu, E. S. Kong, Y. Zhang and S. T. Lee, "Nanowelded carbon-nanotube-based solar microcells", Small 4(9), 1313-1318 (2008). http://dx.doi. org/10.1002/smll.200701309

[4] K. Nanda, A. Maisels, F. Kruis, H. Fissan and S.Stappert, "Higher surface energy of free nanoparticles", Phys. Rev. Lett. 91(10), 106102-106105 (2003). http://dx.doi.org/10.1103/PhysRevLett. 91.106102

[5] S. J. Kim and D. J. Jang, "Laser-induced nanowelding of gold nanoparticles", Appl. Phys. Lett. 86(3), 033112.1-033112.3 (2005). http://dx.doi.org/ 10.1063/1.1856139

[6] H. Tohmyoh, T. Imaizumi, H. Hayashi and M. Saka, "Welding of Pt nanowires by Jouleheating", Scr. Mater. 57(10), 953-956 (2007). http://dx.doi.org/ 10.1016/j.scriptamat. 2007.07.018

[7] K. Asaka, M. Karita and Y. Saito, "Joining of multiwall carbon nanotubes for the end-contact configuration by applying electric current", Mater. Lett. 65(12), 1832-1834 (2011). http://dx.doi.org/10. 1016/j.matlet.2011.03.081

[8] Q. Yang, S. Bai, G. Wang and J. Bai, "Local recon struction and controllable nanospot welding of multiwalled carbon nanotubes under mild electron beamirradiation", Mater. Lett. 60(20), 2433-3437. http:// dx.doi.org/10.1016/j.matlet.2006.01.071

[9] E. Ide, S. Angata, A. Hirose and K. F. Kobayashi, "Metal-metal bonding process using Ag metalloorganic nanoparticles", Acta. Mater. 53(8), 2385-2393 (2005). http://dx.doi.org/10.1016/j.actamat. 2005.01.047

[10] S. Besner and M. Meunier, "Femtosecond laser synthesis of $\mathrm{AuAg}$ nanoalloys: photoinduced oxidation and ions release", J. Phys. Chem. C 114(23), 
10403-10409 (2010). http://dx.doi.org/10.1021/ jp102461u

[11] V. Amendola and M. Meneghetti, "Laser ablation synthesis in solution and size manipulation of noble metal nanoparticles", Phys. Chem. Chem. Phys. 11(20), 3805-3821 (2009). http://dx.doi.org/10. 1039/b900654k

[12] A. V. Kabashin and M. Meunier, "Synthesis of colloidal nanoparticles during femtosecond laser ablation of gold in water", J. Appl. Phys. 94(12), 7941-7943 (2003). http://dx.doi.org/10.1063/1.1626793

[13] J. Zhang, J. Worley, S. Denommee, C. Kingston, Z. J. Jakubek, Y. Deslandes, M. Post, B. Simard, N. Braidy and G. A. Botton, "Synthesis of metal alloy nanoparticles in solution by laser irradiation of a metal powder suspension", J. Phys. Chem. B 107(29), 69206923 (2003). http://dx.doi.org/10.1021/jp027269k

[14] K. Torigoe, Y. Nakajima and K. Esumi, "Preparation and characterization of colloidal silverplatinumalloys", J. Phys. Chem. 97(31), 8304-8309 (1993). http://dx.doi.org/10.1021/j100133a029

[15] A. Hu, J. Y. Guo, H. Alarifi, G. Patane, Y. Zhou, G. Compagnini and C. X. Xu, "Low temperature sintering of Ag nanoparticles for flexible electronicspack- aging", Appl. Phys. Lett. 97, 153117-153119 (2010).

http://dx.doi.org/+10.1063/1.3502604

[16] R. Prasher, P. Bhattacharya and P. E. Phelan, "Thermal conductivity of nanoscale colloidal solutions (nanofluids)", Phys. Rev. Lett. 94(2), 25901-25904 (2005). http://dx.doi.org/10.1103/ PhysRevLett. 94.025901

[17] S. P. Jang and S. U. S. Choi, "Effects of various parameters on nanofluid thermal conductivity", J. Heat Transfer 129(5), 617-623 (2006). http://dx.doi. org/10.1115/1.2712475

[18] L. Liu, L. Xiao, J. Feng, L. Li, S. Esmaeili and Y. Zhou, "Bonding of immiscible $\mathrm{Mg}$ and $\mathrm{Fe}$ by coated nanoscale $\mathrm{Fe}_{2} \mathrm{Al}_{5}$ transition layer", Scr. Mater. 65(11), 982-985 (2011). http://dx.doi.org/10.1016/ j.scriptamat. 2011.08.026

[19] A. Hu, Y. Zhou and W. W. Duley, "Femtosecond laser-induced nanowelding: fundamentals and applications", Open Surf. Sci. J. 3, 42-45 (2011).

[20] W. Hu, S. Xiao, J. Yang and Z. Zhang, "Melting evolution and diffusion behavior of vanadiumnanoparticles", Eur. Phy. J. B 45(4), 547-554 (2005). http://dx.doi. org/10.1140/epjb/e2005-00210-8 